\title{
AVALIAÇÃO DE GENÓTIPOS DE TOMATEIRO TIPO SANTA CRUZ NO PERÍODO DE INVERNO, EM ARAGUARI, MG ${ }^{1}$
}

\author{
JOSÉ RICARDO PEIXOTO², CARLOS MANOEL DE OLIVEIRA ${ }^{3}$, ROGÉRIO PEREIRA DA SILVA ${ }^{4}$, \\ BRUNO DE ANGELIS ${ }^{5}$ e ARTHUR BERNARDES CECÍLIO FILHO ${ }^{6}$
}

\begin{abstract}
RESUMO - Desenvolveu-se um trabalho de pesquisa na fazenda Jordão (município de Araguari, MG), na época de inverno, com o objetivo de verificar o desempenho agronômico de genótipos de tomateiro tipo Santa Cruz. Utilizou-se o delineamento experimental de blocos casualizados, com 14 tratamentos (genótipos) e quatro repetições. A parcela experimental foi constituída por duas fileiras com 12 plantas cada, no espaçamento de $1,00 \mathrm{~m}$ entre linhas e $0,60 \mathrm{~m}$ entre plantas (duas plantas por cova). As colheitas iniciaram-se em 25/8/96 e encerraram em 19/10/96, perfazendo um total de 17 . De forma geral, todos os genótipos apresentaram produtividades elevadas, comparativamente ao rendimento médio nacional, principalmente os genótipos Santa Clara I-5300, Débora Plus, Santa Clara, Santa Clara Importada e Santa Clara III, todos com produtividade superior a $125 \mathrm{t} / \mathrm{ha}$, podendo ser recomendados para plantio na região, no período de inverno. Apenas o genótipo Tom-556 e a cultivar Ângela Gigante I-5100 não tiveram peso médio de frutos superior a 100 g. As cultivares Saladinha, Santa Clara Importada, Concord e Jumbo, tiveram peso médio superior a $130 \mathrm{~g}$, os quais se destacaram, também, em porcentagem de frutos tipo extra AA, juntamente com Santa Clara, Santa Clara I-5300, IAC Santa Clara, enquanto os genótipos Tom-556, Tex-015 e Ângela Gigante I-5100, se destacaram em frutos tipo extra A.
\end{abstract}

Termos para indexação: tomate, Lycopersicon esculentum, produtividade, qualidade

\section{EVALUATION OF TOMATO GENOTYPES (SANTA CRUZ TYPE) DURING THE WINTER SEASON, IN ARAGUARI, MG, BRAZIL}

\begin{abstract}
A field experiment was carried out in the Jordão farm in Araguari, MG, Brazil, during the winter season, to evaluate the agronomic potential of genotypes (Santa Cruz type) of tomato. The experimental design was in randomized blocks with 14 treatments (genotypes) and four replications. The experimental plot was made up of two lines $(1.0 \mathrm{~m}$ between lines and $0.6 \mathrm{~m}$ between plants) with 12 plants each (two plants/pit). Seventeen harvests were made, starting in $8 / 25 / 96$, and ending in $10 / 19 / 96$. In general, all genotypes showed high production compared to the Brazilian production average. The genotypes Santa Clara I-5300, Débora Plus, Santa Clara, Santa Clara (Imported) and Santa Clara III showed production higher than 125 tons/ha, indicating that those genotypes can be cultivated in that region, in the winter season. Only two genotypes, Tom-556 and Ângela Gigante I-5100 did not show fruit weight higher than $100 \mathrm{~g} /$ fruit. The cultivars Saladinha, Santa Clara (Imported), Concord and Jumbo showed average weight higher than $130 \mathrm{~g} /$ fruit, and high percentage of fruits classified as extra AA type; Santa Clara, Santa Clara I-5300, IAC Santa Clara, while Tom-556, Tex-015 and Ângela Gigante I-5100 presented higher number of fruits type A.
\end{abstract}

Index terms: tomato, Lycopersicon esculentum, productivity, quality.

\footnotetext{
${ }^{1}$ Aceito para publicação em 26 de março de 1999.

${ }^{2}$ Eng. Agr., Dr., Faculdade de Agronomia e Medicina Veterinária, Universidade de Brasília (UnB), Caixa Postal 04508, CEP 70910-900Ｂrasília, DF. E-mail: peixoto@unb.br

${ }^{3}$ Eng. Agr., Mestrando em Agronomia, Universidade Federal de Lavras, Caixa Postal 37, CEP 37200-000 Lavras, MG.
}

\footnotetext{
${ }^{4}$ Eng. Agr., Mestrando em Agronomia, UnB.

${ }^{5}$ Estudante de Agronomia, Universidade Federal de Uberlândia, Caixa Postal 593, CEP 38400-902 Uberlândia, MG.

${ }^{6}$ Eng. Agr., Dr., Faculdade de Agronomia, UNESP, Rod. Paulo Donato Castellani, km 5, s/no, CEP 14870-000 Jaboticabal, SP
} 


\section{INTRODUÇÃO}

O potencial genético da semente, aliado à qualidade física, fisiológica e sanitária, são fatores que determinam, em grande parte, o sucesso da tomaticultura, considerada, atualmente, como a segunda hortaliça cultivada no Brasil.

A seleção de cultivares, híbridos ou variedades de polinização aberta, mais produtivos e geneticamente resistentes a patógenos e pragas, constitui uma alternativa ideal para o desenvolvimento tecnológico da cultura. Tais cultivares representam soluções duradouras para certos problemas e são acessíveis à maioria dos agricultores (Ferraz \& Mendes, 1992).

A avaliação de cultivares dentro das mesmas condições edafoclimáticas permite comparar genótipos quanto ao seu potencial de produtividade, qualidade de frutos e resistência a doenças e pragas, e é importante para se conhecer os mais adequados para cada região, pois cada material tem suas características genéticas que determinam sua maior ou menor sensibilidade às condições ambientais e a outros fatores de produção.

Ensaios com avaliação de características agronômicas têm oferecido importantes contribuições no que diz respeito às adaptações edafoclimáticas das cultivares com potencial, nas diversas regiões do Brasil (Leal, 1973; Silva, 1996).

O uso de cultivares pouco adaptadas pode resultar em perda de rendimento e qualidade dos frutos, maior suscetibilidade às doenças e pragas, degenerescência, distúrbios fisiológicos, baixa capacidade de conservação, manipulação e transporte e/ou na obtenção de produtos atípicos em relação às preferências do consumidor. As variações que ocorrem no comportamento de cultivares de tomate introduzidas a partir de outros estados ou países são atribuídas principalmente ao efeito ambiental, em especial às condições edafoclimáticas distintas entre os locais em que foi obtida e/ou selecionada a cultivar e aquele onde foi cultivada. Todas as demais tecnologias são ineficientes quando não se utiliza a cultivar adequada à região de cultivo (Silva Júnior et al., 1995).

Conduziu-se este trabalho com o objetivo de avaliar agronomicamente 14 genótipos de tomate do tipo Santa Cruz, no período de inverno, em Araguari, MG, que se destaca por ser importante região agrícola do País.

Pesq. agropec. bras., Brasília, v.34, n.12, p.2247-2251, dez. 1999

\section{MATERIALE MÉTODOS}

$\mathrm{O}$ experimento foi instalado e conduzido na fazenda Jordão, localizada no município de Araguari, MG, na época do inverno (transplante de mudas em 25/5/96 e última colheita em 19/10/96)

O município está localizado a $18^{\circ} 38^{\prime} 30^{\prime \prime}$ de latitude sul e 48 $11^{\prime} 18^{\prime \prime}$ de longitude oeste de Greenwich. Apresenta uma altitude que varia de $505 \mathrm{~m}$ a $1.087 \mathrm{~m}$ e temperatura média anual de $20,7^{\circ} \mathrm{C}$, sendo a média mínima anual de $16,0^{\circ} \mathrm{C}$, e a média máxima anual de $26,3^{\circ} \mathrm{C}$. A precipitação média anual é de $1.641 \mathrm{~mm}$.

Foram utilizadas as variedades: Ângela Gigante I-5100, Concord, Jumbo AG-592, Santa Clara I-5300, IAC Santa Clara, Santa Clara, Santa Clara Importada, Santa Clara III e Tom-556 e os híbridos: Cláudia, Débora Plus, Saladinha, Bruna e Tex-015.

A adubação foi realizada segundo as recomendações da Comissão de Fertilidade do Solo do Estado de Minas Gerais (1989) e de acordo com a análise química do solo que apresentou os seguintes teores: $\mathrm{pH}$ (água) $=6,0$; $\mathrm{P}=4,0 \mathrm{mg} / \mathrm{dm}^{3 ;}, \mathrm{K}=160 \mathrm{mg} / \mathrm{dm}^{3} ; \mathrm{Al}=0,0 \mathrm{cmol} / \mathrm{dm}^{3}$; $\mathrm{Ca}=4,6 \mathrm{cmol} / \mathrm{dm}^{3} ; \mathrm{Mg}=1,4 \mathrm{cmol} / \mathrm{dm}^{3} ; \mathrm{H}+\mathrm{Al}=2,6 \mathrm{cmol} / \mathrm{dm}^{3}$; $\mathrm{SB}($ Soma de Bases $)=6,4 \mathrm{cmol} / \mathrm{dm}^{3} ; \mathrm{t}(\mathrm{CTC}$ efetiva $/ \mathrm{CTC}$ a $\mathrm{pH} 7,0)=6,0 \mathrm{cmol} / \mathrm{dm}^{3} ; \mathrm{T}=9,0 \mathrm{cmol} / \mathrm{dm}^{3} ; \mathrm{V}=71 \% ; \mathrm{m}$ $($ Sat. de $\mathrm{Al})=0 \%$ e matéria orgânica - M.O. (WalkleyBlack) $=2,9 \mathrm{dag} / \mathrm{kg}$

No plantio, em cada metro linear de sulco, foram utilizados $2 \mathrm{~kg}$ de esterco de curral curtido, $300 \mathrm{~g} \mathrm{de}$ superfosfato simples, $30 \mathrm{~g}$ de cloreto de potássio e $10 \mathrm{~g}$ de FTE.

Realizaram-se quatro adubações de cobertura, via solo, com intervalo de 14 dias, utilizando-se $25 \mathrm{~g} /$ planta de sulfato de amônio, $25 \mathrm{~g}$ /planta de nitrocálcio e $15 \mathrm{~g}$ /planta de cloreto de potássio, e foliar (Orgamin a 0,02\% e Ouro Verde a $0,01 \%$ ) também com intervalo de 14 dias. As sementes foram obtidas junto a empresas especializadas. As mudas foram formadas em bandejas de isopor tipo "spedelling" com 128 células, contendo substrato composto por vermiculita e casca de Pinus sp. Utilizou-se o espaçamento $1,0 \times 0,6 \mathrm{~m}$ com duas plantas/cova, sendo as plantas conduzidas no sistema de tutoramento de cerca cruzada, com uma haste por planta.

Realizaram-se pulverizações preventivas e curativas semanais, com fungicidas e/ou bactericidas e inseticidas, visando ao controle de patógenos e pragas. Foram também efetuados todos os tratos culturais indispensáveis à cultura. 
Utilizou-se o delineamento experimental de blocos casualizados, com quatro repetições e 14 tratamentos (14 genótipos); cada parcela foi constituída de 24 plantas Foram avaliados, num total de 17 colheitas, os seguintes parâmetros: produtividade comercial, peso médio de frutos, número de frutos por planta, porcentagem de frutos tipo extra AA, extra A e primeira. Tal classificação foi feita de acordo com o mercado local, baseando-se na aparência e no tamanho do fruto.

A primeira colheita foi efetuada aos 92 dias após o transplante das mudas, prolongando-se por oito semanas, realizando-se duas colheitas por semana.

As análises empregadas para avaliação dos resultados foram baseadas em modelo apropriado para o delineamento utilizado, de acordo com Pimentel-Gomes (1978). As médias foram comparadas entre si, pelo teste de Tukey, a $5 \%$ de probabilidade

\section{RESULTADOS E DISCUSSÃO}

De forma geral, todos os genótipos testados apresentaram bom desempenho, com produtividade comercial média de 119 t/ha; não houve diferença significativa entre 13 dos 14 genótipos testados (Tabela 1). Em experimento realizado próximo ao local, no período de verão, Silva (1996) verificou maior produtividade comercial da cultivar Saladinha, com 79,2 t/ha; a cultivar Santa Clara I-5300 produziu apenas 51,3 t/ha. Silva Júnior et al. (1995) verificaram maior produção comercial das cultivares Débora, Jumbo, Santa Clara e Cláudia, com rendimentos acima de 72 t/ha em experimento realizado no município de Itajaí, SC.

Em termos de qualidade de frutos, destacaramse sete genótipos, especialmente Santa Clara Importada e Saladinha $F_{1}$. Na produção de frutos do tipo extra A e primeira, destacaram-se as variedades Ângela Gigante I-5100, Tom-556 e Tex-015 F (Tabela 2). No verão, Silva (1996) obteve melhor qualidade da cultivar Saladinha, com $64,2 \%$ de frutos do tipo extra AA e 63,3\% na cultivar Atlas, enquanto Santa Clara Importada apresentou 48,5\% de frutos do tipo extra AA, superada por Concord $(52,3 \%)$, Santa Clara III $(50,1 \%)$ e Santa Clara I-5300 (48,9\%). Em Santa Catarina, Silva Júnior et al. (1995) verificaram maior uniformidade de produção de frutos graúdos nas cultivares Débora e Jumbo.

Aproximadamente 79\% dos genótipos apresentaram peso médio dos frutos superior a $112 \mathrm{~g}$, com destaque para as variedades Saladinha, Jumbo,

TABELA 1. Produção agronômica de 14 genótipos de tomate tipo Santa Cruz, no período de inverno, em Araguari, MG, 1996 ${ }^{1}$

\begin{tabular}{lccc}
\hline Genótipo & $\begin{array}{c}\text { Produtividade } \\
\text { comercial (t/ha) }\end{array}$ & $\begin{array}{c}\text { Peso médio de } \\
\text { frutos (g) }\end{array}$ & $\begin{array}{c}\text { Número de } \\
\text { frutos/planta }\end{array}$ \\
\hline Santa Clara I-5300 & $135,23 \mathrm{a}$ & $126,87 \mathrm{ab}$ & $62,00 \mathrm{ab}$ \\
Débora Plus & $133,58 \mathrm{ab}$ & $118,07 \mathrm{abc}$ & $69,75 \mathrm{a}$ \\
Santa Clara & $130,05 \mathrm{ab}$ & $121,99 \mathrm{abc}$ & $61,50 \mathrm{ab}$ \\
Santa Clara Importada & $128,85 \mathrm{ab}$ & $131,19 \mathrm{a}$ & $58,75 \mathrm{abc}$ \\
Santa Clara III & $125,80 \mathrm{ab}$ & $118,74 \mathrm{abc}$ & $62,75 \mathrm{ab}$ \\
Cláudia & $124,16 \mathrm{ab}$ & $117,82 \mathrm{abc}$ & $65,00 \mathrm{ab}$ \\
Concord & $119,35 \mathrm{ab}$ & $130,50 \mathrm{a}$ & $55,00 \mathrm{bc}$ \\
Bruna & $118,66 \mathrm{ab}$ & $112,58 \mathrm{abc}$ & $63,25 \mathrm{ab}$ \\
Saladinha & $114,63 \mathrm{ab}$ & $134,83 \mathrm{a}$ & $48,00 \mathrm{c}$ \\
IAC Santa Clara & $112,82 \mathrm{ab}$ & $112,83 \mathrm{abc}$ & $55,25 \mathrm{bc}$ \\
Jumbo AG-592 & $111,57 \mathrm{ab}$ & $131,54 \mathrm{a}$ & $54,75 \mathrm{bc}$ \\
Tom-556 & $108,87 \mathrm{ab}$ & $96,07 \mathrm{c}$ & $67,00 \mathrm{ab}$ \\
Tex-015 & $107,53 \mathrm{ab}$ & $102,19 \mathrm{bc}$ & $65,75 \mathrm{ab}$ \\
Ângela Gigante I-5100 & $104,91 \mathrm{~b}$ & $99,34 \mathrm{c}$ & $64,50 \mathrm{ab}$ \\
\hline CV (\%) & 9,86 & 9,16 & 8,47 \\
\hline${ }^{1}$ Médias seguidas pelas mesmas letras, nas colunas, não diferem estatisticamente entre si, pelo teste de Tukey, a $5 \%$ de probabilidade.
\end{tabular}


TABELA 2. Frutos tipo extra AA, extra A e primeira, de tomate tipo Santa Cruz, colhidos no período de inverno, em Araguari, MG, $1996^{1}$.

\begin{tabular}{lccc}
\hline Genótipo & $\begin{array}{c}\text { Frutos tipo extra AA } \\
(\%)\end{array}$ & $\begin{array}{c}\text { Frutos tipo extra A } \\
(\%)\end{array}$ & $\begin{array}{c}\text { Frutos tipo primeira } \\
(\%)\end{array}$ \\
\hline Santa Clara I-5300 & $53,25 \mathrm{ab}$ & $35,30 \mathrm{efg}$ & $11,45 \mathrm{~d}$ \\
Débora Plus & $45,78 \mathrm{bc}$ & $44,22 \mathrm{cde}$ & $10,00 \mathrm{~d}$ \\
Santa Clara & $54,25 \mathrm{ab}$ & $33,28 \mathrm{fg}$ & $12,47 \mathrm{~cd}$ \\
Santa Clara Importada & $61,82 \mathrm{a}$ & $28,68 \mathrm{~g}$ & $9,50 \mathrm{~d}$ \\
Santa Clara III & $48,00 \mathrm{bc}$ & $40,35 \mathrm{cdef}$ & $11,65 \mathrm{~d}$ \\
Cláudia & $36,83 \mathrm{c}$ & $46,23 \mathrm{~cd}$ & $16,94 \mathrm{bcd}$ \\
Concord & $51,45 \mathrm{ab}$ & $35,85 \mathrm{efg}$ & $12,70 \mathrm{~cd}$ \\
Bruna & $35,60 \mathrm{c}$ & $48,08 \mathrm{bc}$ & $16,32 \mathrm{bcd}$ \\
Saladinha & $61,07 \mathrm{a}$ & $29,35 \mathrm{~g}$ & $9,58 \mathrm{~d}$ \\
IAC Santa Clara & $55,28 \mathrm{ab}$ & $33,52 \mathrm{fg}$ & $11,20 \mathrm{~d}$ \\
Jumbo AG-592 & $50,88 \mathrm{ab}$ & $37,95 \mathrm{defg}$ & $11,17 \mathrm{~d}$ \\
Tom-556 & $21,00 \mathrm{~d}$ & $57,85 \mathrm{a}$ & $21,15 \mathrm{abc}$ \\
Tex-015 & $17,15 \mathrm{~d}$ & $56,40 \mathrm{ab}$ & $26,45 \mathrm{a}$ \\
Ângela Gigante I-5100 & $16,05 \mathrm{~d}$ & $58,75 \mathrm{a}$ & $25,20 \mathrm{ab}$ \\
\hline CV (\%) & 11,38 & 9,09 & 24,34 \\
\hline 1
\end{tabular}

${ }^{1}$ Médias seguidas pelas mesmas letras, nas colunas, não diferem estatisticamente entre si, pelo teste de Tukey, a $5 \%$ de probabilidade.

Santa Clara Importada e Concord, que ultrapassaram $130 \mathrm{~g} /$ fruto, enquanto Ângela Gigante I-5100 e Tom-556 apresentaram peso médio inferior a $100 \mathrm{~g} /$ fruto (Tabela 2). Silva (1996) verificou peso médio superior a $110 \mathrm{~g}$ em $75 \%$ dos genótipos avaliados. Silva Júnior et al. (1995) verificaram menor peso médio de frutos da cultivar Débora, em relação aos materiais mais promissores, embora tenha sido a cultivar mais produtiva para as condições edafoclimáticas do litoral de Itajaí, SC.

O híbrido Débora Plus apresentou o maior número de frutos comerciais/planta $(69,75)$, seguida pelas variedades Tom-556 $(67,00)$, Tex-015 $(65,75)$ e Cláudia $(65,00)$. Entretanto os genótipos Tom-556 e Tex-015 apresentaram frutos de menor tamanho, em comparação aos demais materiais. No verão, Silva (1996) verificou maior número de frutos/planta nas cultivares Débora Plus $(32,10)$ e Saladinha $(30,10)$.

Houve correlação positiva entre a produtividade comercial e o peso médio de frutos $(r=0,52)$ e também com a porcentagem de frutos tipo extra AA $(r=0,60)$. Por outro lado, obteve-se correlação negativa entre a produtividade comercial e a porcentagem de frutos tipo extra A $(r=-0,52)$, e também com a porcentagem de frutos tipo primeira $(r=-0,65)$, deduzindo-se que a produtividade foi altamente dependente da eficiência da planta quanto à absorção e utilização de água, nutrientes e outros fatores de produção, e pouco dependente da sua capacidade de produção de flores, do abortamento e do número de frutos.

A diminuição do tamanho dos frutos com o aumento do número de frutos por planta ficou bem evidente, pois houve correlação negativa entre número de frutos/planta, com prejuízo para a sua classificação.

\section{CONCLUSÕES}

1. De modo geral, todos os genótipos apresentam produtividades elevadas, comparativamente ao rendimento médio nacional.

2. Os híbridos não superam agronomicamente a tradicional cultivar Santa Clara.

\section{AGRADECIMENTOS}

Às empresas que forneceram as sementes (HortiAgro, Agroceres, Agroflora, Topseed, Isla, Asgrow); aos senhores Rui Alves Peixoto (Produtor Rural), Rui Alves Peixoto Júnior, Joaquim Lopes (Auxiliar 
Técnico da UFU), Rinaldo Alves Peixoto, Vanderlei Batista da Silva e Júnio Batista Carneiro, pela valiosa ajuda na instalação, condução e avaliações do experimento.

\section{REFERÊNCIAS}

COMISSÃO DE FERTILIDADE DO SOLO DO ESTADO DE MINAS GERAIS. Recomendações para o uso de corretivos e fertilizantes em Minas Gerais, 4⿳亠口冋 aproximação. Lavras, 1989. 176p

FERRAZ, F; MENDES, M. de L. O nematóide das galhas. Informe Agropecuário, Belo Horizonte, v.16, n. 172, p.43-45, 1992.
LEAL, N.R. Comparação da produtividade do cultivar de tomate "Alcobaça" com três cultivares do tipo "Santa Cruz", na Baixada Fluminense. Revista Ceres, Viçosa, v.20, n.107, p.65-67, jan./mar. 1973.

PIMENTEL-GOMES, F. Curso de Estatística Experimental. 8.ed. São Paulo: Nobel, 1978. 430p.

SILVA, R.P. da. Avaliação de genótipos de tomate tipo Santa Cruz, no período de verão em Araguari MG. Uberlândia: UFU, 1996. 31p.

SILVA JÚNIOR, A.A.; VIZZOTO, V.J.; STUKER, H Cultivares de tomate para o Baixo Vale do Itajaí. Agropecuária Catarinense, Santa Catarina, v.8, n.4, p.35-39, dez. 1995. 